\title{
INFLUÊNCIA DO USO DA TERRA NA VULNERABILIDADE DOS POÇOS TUBULARES NA ZONA AGRÍCOLA DA DEPRESSÃO DE QUÍBOR NA VENEZUELA
}

\author{
Luís Alfredo Diaz Jaramillo ${ }^{(a)}$, Janete Teresinha Reis ${ }^{(b)}$, Roberta Araujo Madruga ${ }^{(c)}$ \\ (a) Fundação Instituto de Engenharia da Venezuela, ldiazjaramillo@gmail.com \\ (b) Programa de Pós-graduação em Geografia, Universidade Federal de Santa Maria, reis.janete@gmail.com \\ (c) Programa de Pós-graduação em Geografia, Universidade Federal de Santa Maria, robertamadruga@yahoo.com.br
}

\section{Eixo: GEOTECNOLOGIAS E MODELAGEM ESPACIAL EM GEOGRAFIA FÍSICA}

\begin{abstract}
Resumo
O uso da terra interfere diretamente na qualidade da água e na vulnerabilidade dos poços tubulares. Neste sentido, o presente estudo objetivo determinar a influência do uso da terra na vulnerabilidade dos poços tubulares na zona de aproveitamento agrícola da Depressão de Quíbor, no município Jiménez na Venezuela. Para tanto, utilizou-se o método GOD, proposto por Foster e Hirata (1988), para determinar o índice de vulnerabilidade, aliado a interpolação dos poços por meio da Krigagem resultando no mapa de vulnerabilidade. O uso da terra foi analisado a partir da imagem SPOT 5, mediante classificação supervisada gerando como produto final o mapa. Posteriormente, obteve-se a integração dos dois mapas, elaborando o mapa síntese indicando as áreas mais vulneráveis. De acordo com o índice de GOD, constatou-se que a maioria dos poços tubulares apresenta vulnerabilidade de média a moderada em uso agrícola. Sendo assim, o índice de vulnerabilidade favorece o desenvolvimento agrícola.
\end{abstract}

Palavras chave: uso da terra, vulnerabilidade, poços tubulares, área agrícola

\section{Introdução}

O uso da terra tem influência direta nas características físicas da água, tanto superficial como subterrânea. Neste sentido, a interferência da ação antrópica a partir de atividades, como por exemplo, industriais e agrícolas afetam negativamente as propriedades da água, acarretando na contaminação dos recursos hídricos.

A água como recurso natural é vital para o sustento dos seres vivos, bem como sua importância nas atividades agrícolas e industriais. É de fundamental relevância o estudo do meio aquático para determinar a influência que o ser humano exerce sobre esse importante recurso. A crescente contaminação das águas superficiais por causa de indústrias, expansão desordenada da população urbana e uso excessivo de agroquímicos vêm desencadeando consequências negativas no meio ambiente. Este fato tem gerado preocupação por parte dos órgãos governamentais visando à criação de leis que previnam a deterioração dos recursos naturais. 


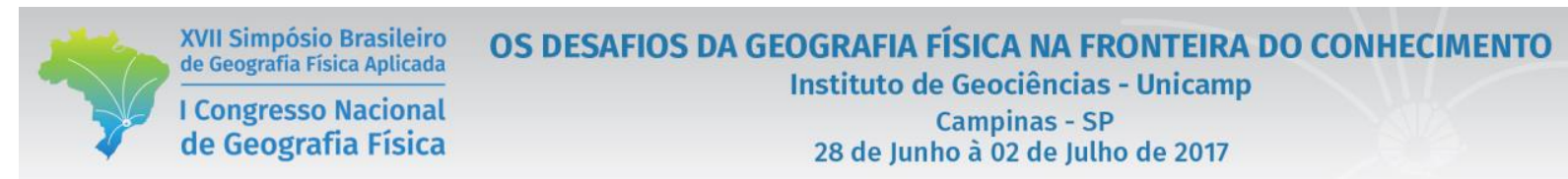

Segundo Otálvaro (1999), as reservas de água a nível global se encontram distribuídas em 97,2\% de água salgada e $2,8 \%$ de água doce. Desta última, somente $0,6 \%$ é agua subterrânea da qual apenas $0,3 \%$ podem ser exploradas para o consumo humano pela sua viabilidade econômica, o que demonstra a grande importância dessas águas.

As águas subterrâneas são formadas pela precipitação que infiltra direta ou indiretamente na superfície do solo. Devido a isto, a influência antrópica sobre a qualidade das águas por causa das atividades agrícolas, urbanas e industriais não controladas, excedem a capacidade natural do subsolo e extratos subjacentes por diminuir os efeitos contaminantes destas atividades (BANCO MUNDIAL, 2003). Sendo assim, este estudo tem como objetivo analisar a influência do uso da terra na vulnerabilidade dos poços tubulares da zona de aproveitamento agrícola da depressão de Quíbor na Venezuela.

\section{Metodologia}

\section{1 Área de Estudo}

A área em análise localiza-se no Estado de Lara, mais precisamente no município de Jimenez, na zona do vale de Quíbor na Venezuela (Figura 1). Esta área encontra-se entre as coordenadas $417817 \mathrm{mN}$ a $449817 \mathrm{mN}$ e as coordenadas $1083710 \mathrm{mE}$ a $1115710 \mathrm{mE}$, no fuso 19, totalizando 43.395 hectares.

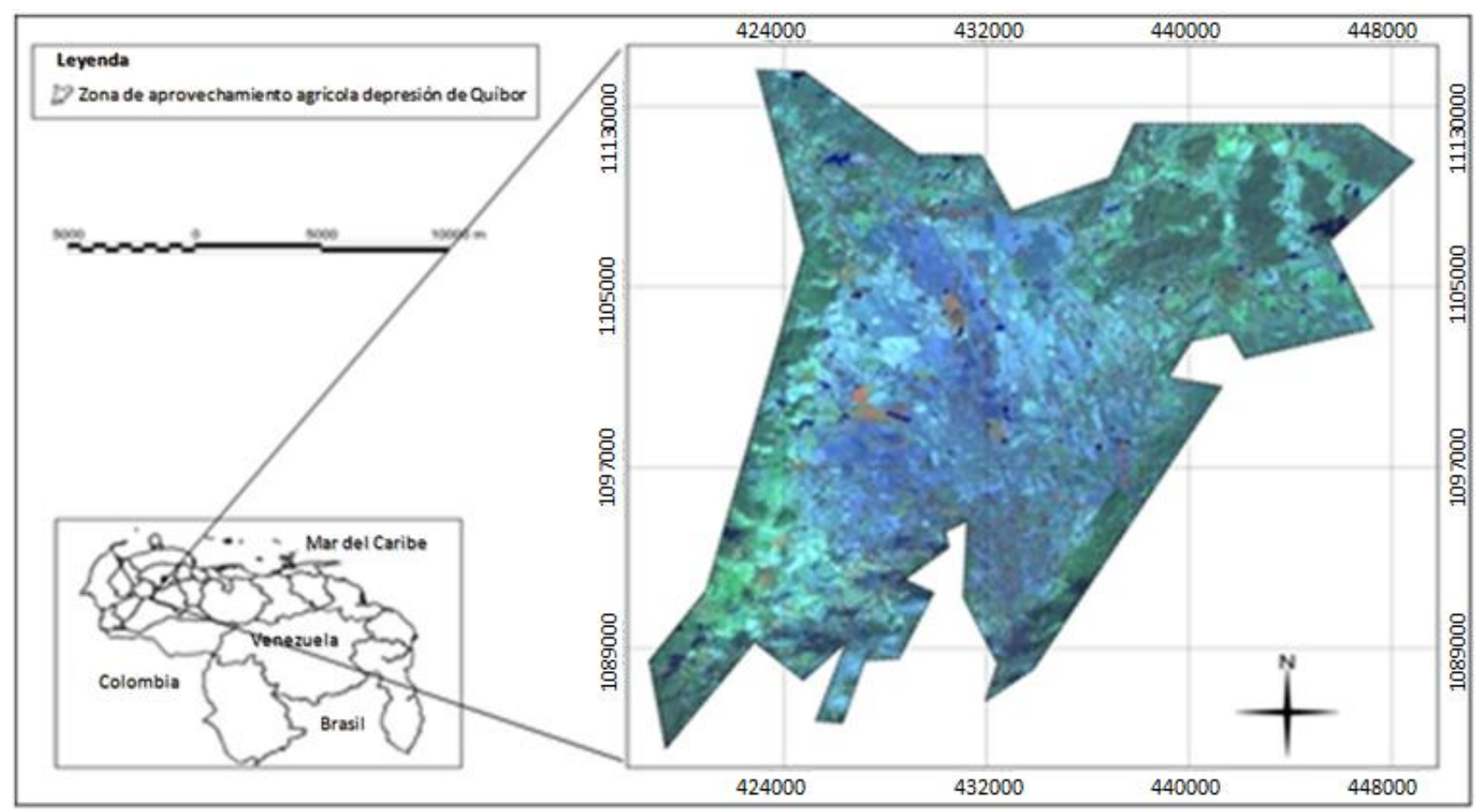

Figura 1 - Localização da área de estudo 


\subsection{Uso da terra}

O mapa de uso da terra foi elaborado utilizando como base uma imagem de satélite SPOT5, com 10 metros de resolução espacial, de 28-01-2012, que foi adquirida pela Fundação Instituto de Engenharia da Venezuela. Foi realizada a classificação supervisionada no software SPRING versão 5.2.1. Para a classificação foi utilizado o algoritmo de Bhattacharya com 99\% de aceitação, para o qual, se obtiveram 100 amostras para cada classe das quais 50 foram de prova. Foram definidas seis classes temáticas: área urbana, agricultura, vegetação, solo exposto, água e nuvens.

\section{3 Índice de vulnerabilidade}

Para o cálculo do índice de vulnerabilidade dos poços tubulares, foi utilizado o método GOD, desenvolvido por Foster e Hirata e adaptado por Foster et al. (2006). Este método se baseia na classificação de índices entre 0 e 1 para determinar a vulnerabilidade de um aquífero quanto a sua contaminação, usando três variáveis que formam a sigla $\mathbf{G}$ (Grau de confinamento hidráulico), $\mathbf{O}$ (Ocorrência de estratos de cobertura litológica) e D (Distância do nível da água subterrânea).

Uma vez estabelecidos os parâmetros necessários para o cálculo do índice de vulnerabilidade foi criado um algoritmo na linguagem de programação PHP5 para o cálculo dos valores de cada poço tubular utilizados pelo método GOD. Para a interpolação dos dados obtidos utilizou-se o método de Krigagem ordinário, calculando a distância máxima entre os pontos para a variação do variograma a partir do software Quantum GIS, gerando o mapa de vulnerabilidade dos poços tubulares da área de estudo.

\subsection{Análise integrada da influência do uso da terra na vulnerabilidade dos poços tubulares}

A análise consistiu em integrar os mapas de índice de vulnerabilidade e o mapa de uso da terra no software Quantum GIS, desta forma podem-se visualizar as classes temáticas do mapa de uso da terra com as classes do mapa de índice de vulnerabilidade.

\section{Análise dos Resultados}

\subsection{Análise do uso da terra}

A área total de estudo compreende 43.409 hectares. Diante da classificação supervisionada foram extraídos os diferentes tipos de uso que correspondem a seis classes: vegetação, agricultura, solo exposto, 
área urbana, água e nuvens (Tabela 1). Esta última mereceu classificação devido sua presença, embora a imagem de satélite selecionada tenha sido a de menor presença de nuvens.

Tabela 1 - Classes de uso da terra na área de estudo

\begin{tabular}{l|r|r|r|r|r|r}
\hline Área & \multicolumn{1}{|c|}{ Água } & \multicolumn{1}{c|}{ Vegetação } & \multicolumn{1}{l|}{ Agricultura } & \multicolumn{1}{l|}{ Solo exposto } & \multicolumn{1}{c}{ Área urbana } & Nuvens \\
\hline Hectares & $1.347,99$ & $14.791,78$ & $22.031,76$ & $3.819,68$ & 870,53 & 547,47 \\
\hline Porcentagem & $3,11 \%$ & $34,08 \%$ & $50,75 \%$ & $8,80 \%$ & $2,01 \%$ & $1,25 \%$ \\
\hline
\end{tabular}

Quanto aos diferentes tipos de uso da terra destaca-se a presença de agricultura, com 50,75\%, seguida da vegetação de $34,8 \%$ e solo exposto com $8,8 \%$. Constatou-se que na área de interesse também se encontra uma área urbana compreendendo em torno de 2\% da área total (Figura 2).

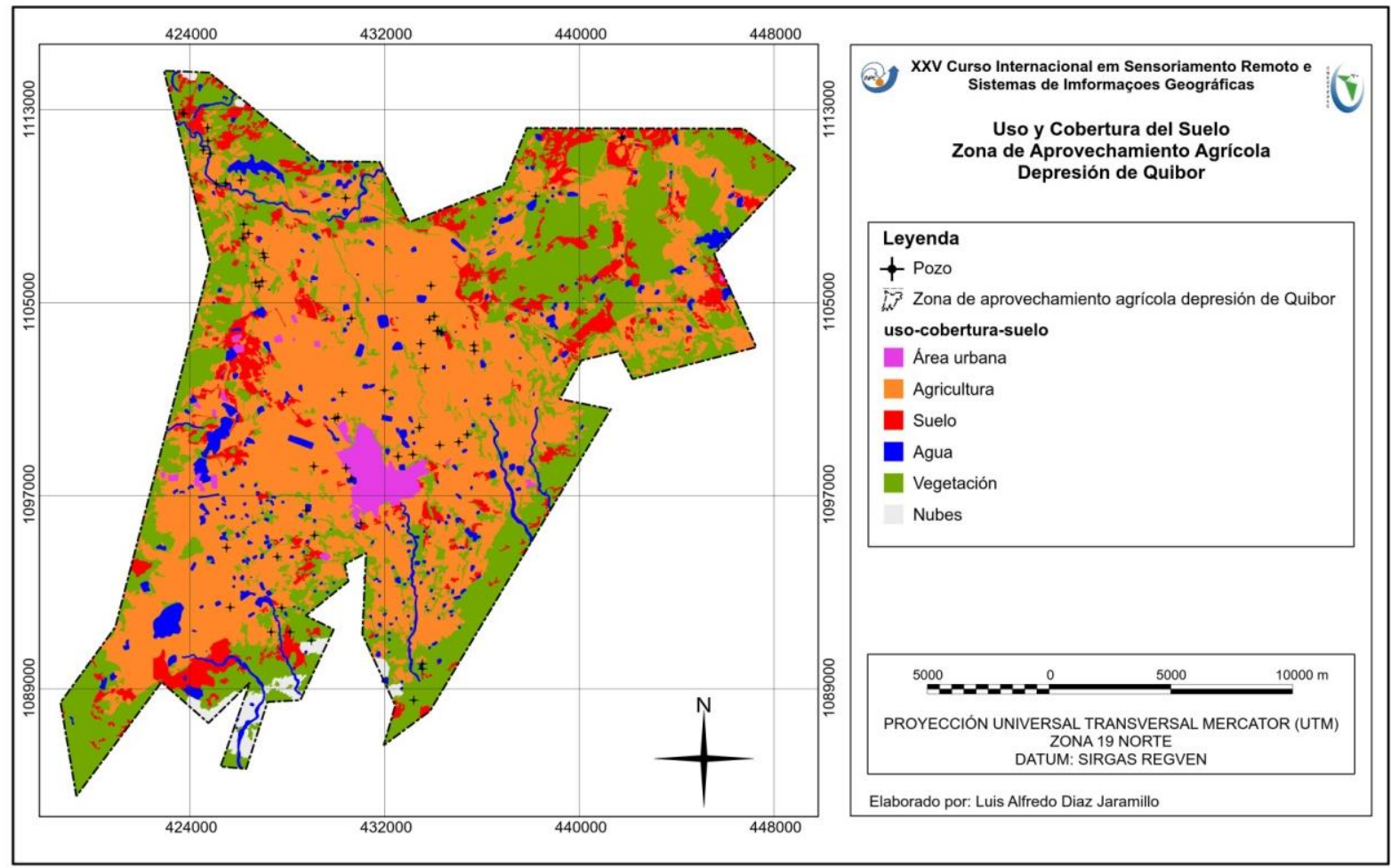

Figura 2 - Mapa de uso da terra para a zona de aproveitamento agrícola da depressão de Quíbor.

Por ser uma área utilizada, principalmente, para o uso agrícola há probabilidade de contaminação difusa. Este tipo de poluição pode ser gerado a partir de resíduos sólidos e de sedimentos, tanto em área rural com urbana. Conforme Porto (1995) e Tomaz (2006; 2007), a poluição difusa além de complexa, provem de diferentes fontes, como: freios de automóveis, resíduos de pneus, resíduos de pinturas em geral, fezes de 


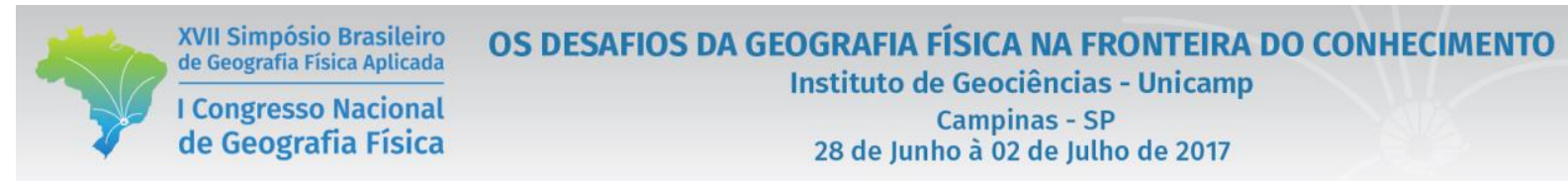

animais, resíduos de ferro, zinco, tanto cobre como alumínio de materiais de construção, restos de vegetação, derramamentos, erosão fuligem, poeira, enxofre, metais, pesticidas, nitritos e nitratos, cloretos, fluoretos silicatos, cinzas, compostos químicos, entre outros. Assim, a área deve ter o constante monitoramento ambiental.

\subsection{Análise da Vulnerabilidade}

Para a análise do índice de vulnerabilidade utilizou-se dados de 59 poços tubulares aplicando-se o método GOD, com os valores compreendidos entre 0,05 e 0,64 e uma média aritmética de 0,07. O resultado da interpolação gerou um mapa com índice de vulnerabilidade com os seguintes valores: 0,0 a 0,1 (desprezível), 0,1 a 0,3 (baixa) e, 0,3 a 0,5 (moderada), onde a maior parte se encontra em áreas de vulnerabilidade desprezível e baixa (Figura 3).

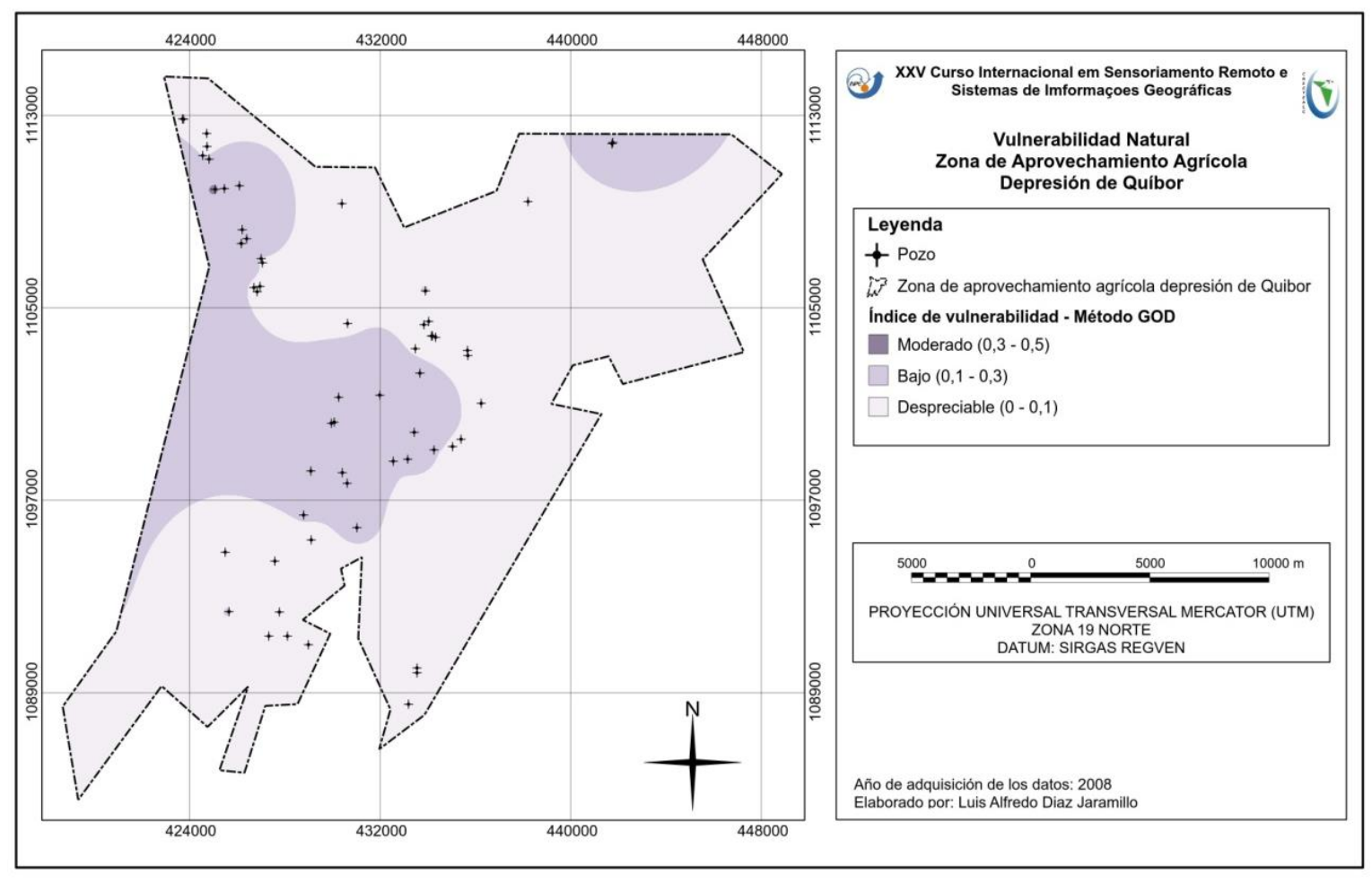

Figura 3 - Mapa de vulnerabilidade dos poços tubulares da zona de aproveitamento agrícola da depressão de Quíbor.

Cabe ressaltar que o índice de vulnerabilidade das águas subterrâneas diverge entre desprezível e moderada, no entanto, a classe moderada identifica poços tubulares mais vulneráveis à contaminação antrópica. 


\begin{tabular}{|c|c|}
\hline $\begin{array}{l}\text { XVII Simpósio Brasileiro } \\
\text { de Geografia Fisica Aplicada }\end{array}$ & $\begin{array}{l}\text { OS DESAFIOS DA GEOGRAFIA FÍSICA NA FRONTEIRA DO CONHECIMENTO } \\
\text { Instituto de Geociências - Unicamp }\end{array}$ \\
\hline $\begin{array}{l}\text { I Congresso Nacional } \\
\text { de Geografia Física }\end{array}$ & $\begin{array}{l}\text { Campinas - SP } \\
28 \text { de Junho à } 02 \text { de Julho de } 2017\end{array}$ \\
\hline
\end{tabular}

\subsection{Análise da influência do uso da terra na vulnerabilidade dos poços tubulares}

$\mathrm{Na}$ zona urbana da área de estudo o índice de vulnerabilidade predominante foi o desprezível, obtendo valores entre 0,0 a 0,1 , somente um pequeno percentual da porção Noroeste da área de estudo possui um índice de vulnerabilidade moderada (Figura 4).

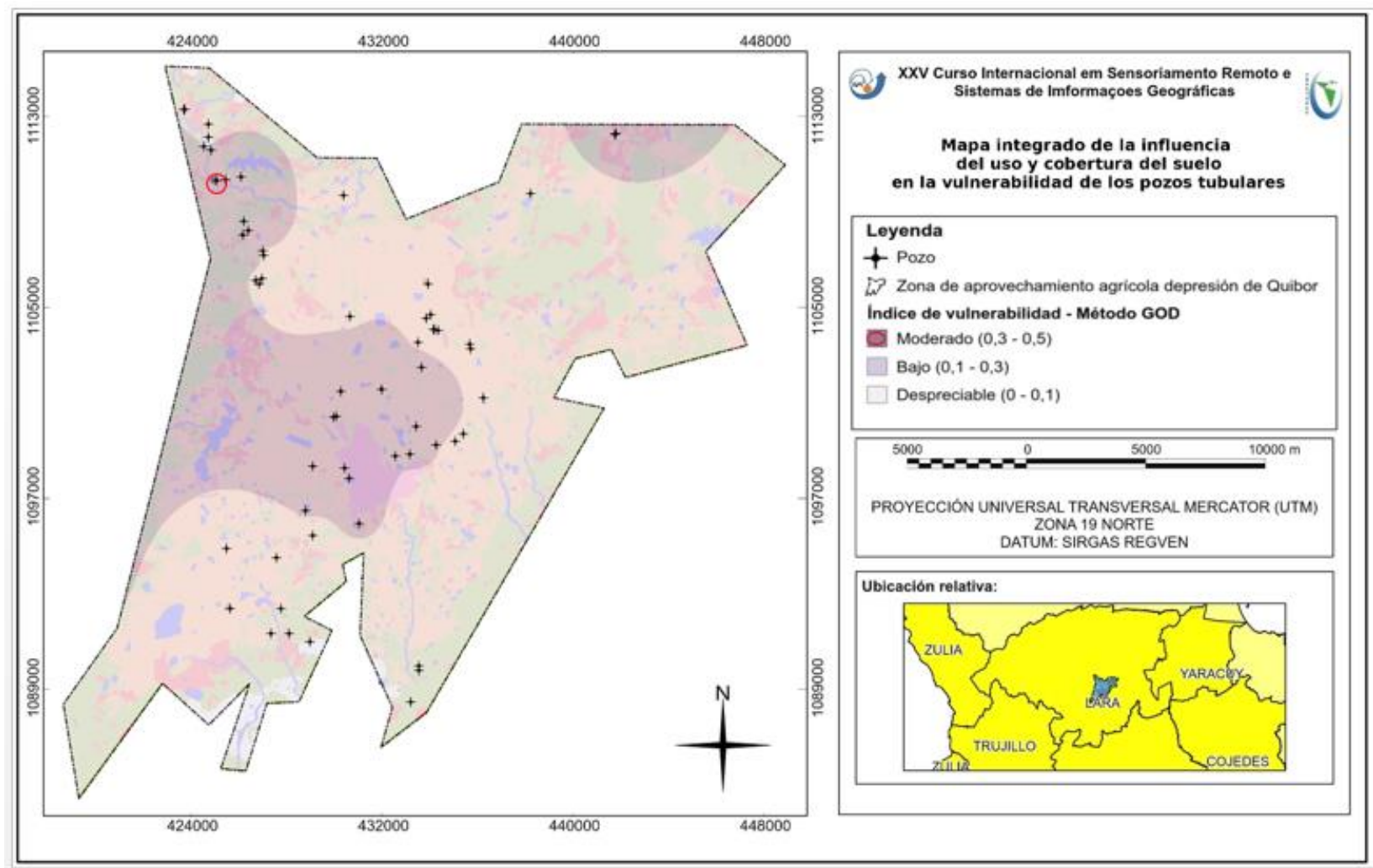

Figura 4 - Influência do uso da terra na vulnerabilidade dos poços tubulares da área de estudo

Portanto, a área moderada indica a maior vulnerabilidade encontrada e neste concentram-se os usos de agricultura e solo exposto entre os índices de 0,3 a 0,5. Na zona de aproveitamento agrícola de depressão de Quíbor não foram encontrados índices altos ou extremos de vulnerabilidade que demandam maiores cuidados para fins de usos agrícolas. Porém, a probabilidade de contaminação pode ocorrer com o aumento deste uso sem os devidos cuidados em virtude de uso de fertilizantes, uma vez que na área agrícola encontra-se a plantação de cebola, aipim, cana de açúcar, entre outros. Deste modo, a água subterrânea que se encontra na área de estudo pode ser utilizada para fins agrícolas. 


\section{Considerações Finais}

Concluiu-se que a maioria dos poços dos tubulares se encontra em zona de aproveitamento agrícola de vulnerabilidade desprezível e baixa, apresentando pequena probabilidade de contaminação. Dessa forma, a análise da vulnerabilidade dos poços tubulares e sua influência nos usos agrícolas são de extrema importância.

Em caso de vulnerabilidade alta ou extrema exigem-se cuidados especiais, tendo em vista que a análise envolve o grau de confinamento hidráulico, a ocorrência de estratos de cobertura litológica e a distância do nível da água subterrânea, que não é o caso da depressão de Quíbor.

A análise da vulnerabilidade pode indicar a possibilidade de contaminação dos poços, em contrapartida, os resultados indicaram que a vulnerabilidade é baixa o que evidencia que o uso da terra para fins agrícolas pode ser explorado, a partir da adoção de métodos adequados de uso.

\section{Bibliografia}

BANCO MUNDIAL. Protección de la calidad del agua subterránea - Guía para empresas de agua, autoridades municipales y autoridades ambientales. 2003.

CUSTODIO E. LLAMAS M. Hidrología Subterránea. Volumen II. Omega. Barcelona, 1983.

DAMBRÓS C., SILVEIRO J., DESCOVI F. L. Mapeamento da vulnerabilidade do aquífero à contaminação no Município de Rosário do Sul - RS, Brasil. XV Simpósio Brasileiro de Sensoriamento Remoto - SBSR, Curitiba, PR, Brasil, 30 de abril a 05 de maio de 2011, INPE p.3875.

FOSTER S., GARDUÑO H., KEMPER K., TUINHOF A., NANNI M., DUMARS C. Protección de la calidad del agua subterránea - Definición de estrategias y establecimiento de prioridades. Banco Mundial. 2006.

FOSTER S., HIRATA R. Groundwater polluttion risk assessment: A methodology using available data. PAN AMERICAN CENTER FOR SANITARY ENGINEERING AND ENVIRONMENTAL SCIENCES, 1988.

HUGUET J. M. Hidrogeologia: Tipos de Acuiferos. Universidad de Santiago de Compostela. Master Universitario en Ingeniería del Agua. Sevilla, 2005. 30 p.

OTÁlVARO V., VICTORIA M. Hidráulica de aguas subterráneas. $2^{\mathrm{a}}$ edición. Universidad Nacional de Colombia, 1999. 


\begin{tabular}{|c|c|}
\hline $\begin{array}{l}\text { XVII Simpósio Brasileiro } \\
\text { de Geografia Fisica Aplicada } \\
\end{array}$ & $\begin{array}{l}\text { OS DESAFIOS DA GEOGRAFIA FÍSICA NA FRONTEIRA DO CONHECIMENTO } \\
\text { Instituto de Geociências - Unicamp }\end{array}$ \\
\hline $\begin{array}{l}\text { I Congresso Nacional } \\
\text { de Geografia Física }\end{array}$ & $\begin{array}{l}\text { Campinas - SP } \\
28 \text { de Junho à } 02 \text { de Julho de } 2017\end{array}$ \\
\hline
\end{tabular}

PORTO, M. F. Aspectos qualitativos do escoamento superficial em áreas urbanas. In: TUCCI, C. E. M; PORTO, Rubem La Laina; BARROS, Mário T. de Barros (Org.). Drenagem Urbana. Porto Alegre: ABRH e Editora da UFRGS, p. 387-414, 1995.

TOMAZ, Plínio. Poluição Difusa. São Paulo: Navegar Editora, 2006.

TOMAZ, Plínio. Material de palestra sobre poluição difusa. 2007 